Defence Science Journal. Vol 51. No 1, January 2001, pp.83-92

(c) 2001. DESIDOC

\title{
Estimation Methods for Determination of Drag Characteristics of Fly-by-Wire Aircraft
}

\author{
$G$ Girija, Basappa, J.R. Raol and Padma Madhuranath \\ National Aerospace Laboratories, Bangalore - 560017
}

\begin{abstract}
In this paper, several parameter/state estimation approachesfor the determination ofdragpolars from flight data are described and evaluated for a fly-by-wire(FBW) aircraft. Bolh model-based approaches (MBAs) and non-model-basedapproaches (NMBAs) are considered. Dynamic response data from roller coaster and windupturn manoeuvres are generated in a FBW aircraft flight simulator at different flight conditionsand the typical performance results are presented. A novel approach to estimate the drag polar has been evaluated. It has been found that the NMBAs perform better than the MBAs .Classically, the MBAs have been used for the determination of drag polars. The merits of an NMBA are that it does not require specification of the detailed model of the aerodynamic coefficients and it can be suitably used for online estimation of drag polars from the flight data of aerospace vehicles.
\end{abstract}

Keywords: Drag polar determination, model-based approaches, non-model-based approaches, fly-by-wireaircraft flight simulator

\section{INTRODUCTION}

Determination of the performance characteristics of an aircraft during flight testing is of great importance'. Systematic evaluation of the drag polars of an aircraft using dynamic manoeuvres can be carried out over the full angle of attack range of the aircraft. In recent years, parameter estimation methods have found extensive use in aircraft applications to determine aircraft performance and stability characteristics using dynamic manoeuvres*.The demands of improved performance characteristics of modern flight vehicles have led to aerodynamically unstable configurations which need to be highly augmented in order that they can be flown. For such an FBW aircraft, parameter estimation and determination of performance characteristics would require special considerations ${ }^{3}$.

In this paper, several state/parameter estimation approaches are compared and evaluated for the first time for drag polar determination using responses generated from a 6-DOF simulator for an FBW aircraft in the country.

Both the model-based approaches (MBAs) and non-model-based approaches (NMBAs) are used for the determination of drag polars. Certain methods have potential application for real-time quick-look drag polar determination. Also, the results of drag polar determination using a novel approach are presented. The latter method does not require knowledge of $\boldsymbol{a}$ priori statistics of process and measurement noises.

\section{PARAMETER/ STATE ESTIMATION METHODS}

The parameter/state estimation methods are linked as shown in Fig. 1. The estimation before modelling (EBM) approach encompasses the NMBA. The main distinction between NMBA and MBA 
is that in the latter the aerodynamic derivatives are explicitly estimated either as direct parameters in stabilised output error method (SOEM) or as additional states in extended $U-D$ filter (EUDF) as shown in Fig. 1 .

\subsection{State \& Measuremeut Models}

The following set of equations are considered:

$$
\begin{aligned}
& x_{j+1}=\phi_{j, j+1} x_{j}+G w_{j} \\
& z_{j}=H x_{j} \mathrm{t} v_{\prime}
\end{aligned}
$$

Here, $\mathbf{x}$ is the state vector, $w$ is the process noise with zero mean and covariance matrix $Q, \mathrm{z}$ is the measurement vector and $\mathrm{v}$ is the measurement noise with zero mean and covariance matrix $R$, all of appropriate dimensions. $\phi$ is the state transition matrix and $H$, the observation model.

\subsection{Basic $U-D$ Filtering Approach}

This filter is implemented in the factorised form for the present application. It is given in two parts: Time propagation algorithm and measurement update algorithm.

\subsection{Time Propagation Algorithm}

State vector evolution (prediction)

$$
\tilde{x}_{j+1}=\phi_{j+1, j} \hat{x}_{j}
$$

Covariance update

$$
\tilde{P}_{j+1}=\phi \hat{P}_{j} \phi^{T} \text { t GQG" }
$$

With $\hat{P}=\hat{U} \hat{D} \hat{U}^{T}$ and covariance matrix Q, the time update factors $\widetilde{U}$ and $\widetilde{D}$ are obtained through modified Gram-Schmidt orthogonalisation process. The matrix $U$ is an upper triangular matrix with unit elements on its main diagonal and $D$ is a diagonal matrix. Covariance and gain processing algorithms, operating on $U$ and $D$ factors of state error covariance matrix $\boldsymbol{P}$, are a technique for implementing 'squareroot filtering' without requiring

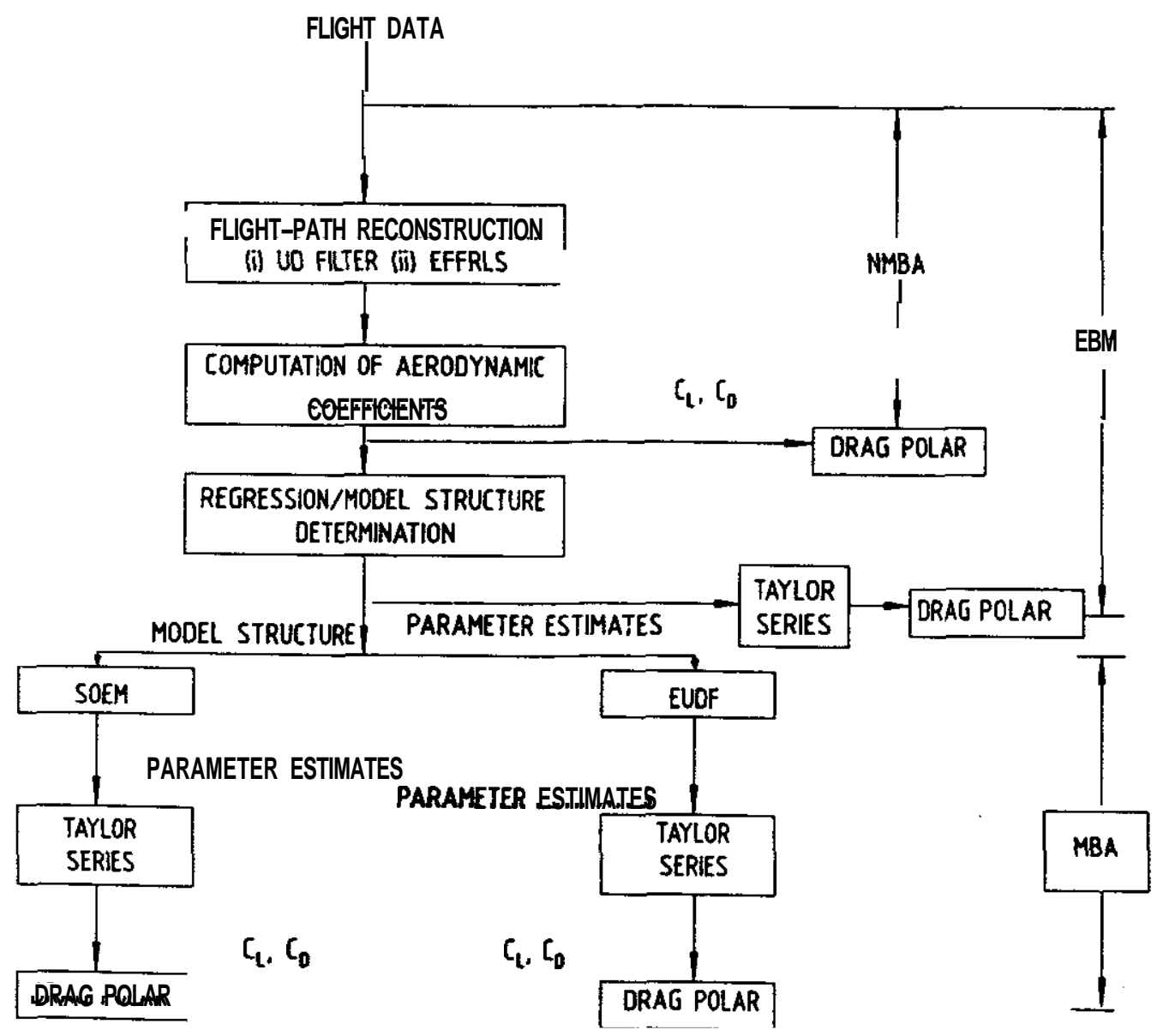

Figure 1. Link between various methods for determination of drag polars from flight data 
computation of square roots. $U \cdot D$ Kalman filtering algorithm is considered efficient, stable and accurate for real-time applications.

One defines

$$
W=[\phi \hat{U} \mid G] ; \quad D=\operatorname{diag}[\hat{D}, Q]
$$

with $W^{\top}=\left[w_{1}, w_{2}, \ldots, w_{n}\right]$,

Here, Tdenotes transpose of vector/matrix. U, $D$ factors of $\widetilde{\boldsymbol{P}}=W D W^{\top}$ may be computed now. For $j=n, n-1, \ldots, 2$, the following equations are recursively evaluated as shown below:

$$
\begin{aligned}
& \tilde{D}_{j}=\left\langle w_{j}^{(n-j)}, w_{j}^{(n-j)}>D\right. \\
& \tilde{U}_{(i, j)}=\left\langle w_{j}^{(n-j)}, w_{j}^{(n-j)}>_{D} / \widetilde{D}_{j} ; i=1, \ldots, j-1\right. \\
& w_{i}^{(n-j+1)}=w_{i}^{(n-j)}-\tilde{U}(i, j) w_{j}^{(n-j)} \\
& \tilde{D}_{1}=\left\langle w_{1}^{(n-1)}, w_{1}^{(n-1)}\right\rangle_{D}
\end{aligned}
$$

Here subscript $D$ qualifies the weighted inner product wrt $\boldsymbol{D}$.

\subsubsection{Measurement Update Algoriihm}

The measurement update in Kalman filtering combines apriori estimate $\tilde{\boldsymbol{x}}$ and error covariance $\tilde{P}$ with scalar observation $\mathrm{z}=a^{T} x+v ; a^{T}=\boldsymbol{H}$ to construct an updated (filtered state) estimate and covariance as follows:

$$
\begin{aligned}
& \mathrm{K}=\tilde{P} a / \alpha \\
& \hat{x}=\tilde{x}+K\left(z-a^{T} \tilde{x}\right) \\
& \mathrm{a}=a^{T} \tilde{P} a i r \\
& \hat{P}=\tilde{P}-K a \tilde{P}
\end{aligned}
$$

Here, $r$ is the measurement noise variance (for scalar data processing). Kalman gain Kand updated covariance factors $\hat{U}$ and $\hat{D}$ can be obtained from the following equations:

$$
\left.\begin{array}{l}
f=\tilde{U}^{T} a, \quad f^{T}=\left(f_{1}, \ldots, f_{n}\right) \\
v=\tilde{D} f ; \quad v_{i}=\tilde{d}_{i} f_{i} \quad ; i=1,2, \ldots, n \\
\hat{d}_{1}=\tilde{d}_{1} r / \alpha_{1} ; \alpha_{1}=r+v_{1} f_{1} ; \\
K_{2}^{T}=\left(v_{1} 0 \ldots 0\right)
\end{array}\right\}
$$

Forj $=2, \ldots, n$ recursively the following equations are evaluated:

$$
\left.\begin{array}{l}
\alpha_{j}=\alpha_{j-1}+v_{j} f_{j} \\
\hat{d}_{j}=\tilde{d}_{j} \alpha_{j-1} / \alpha_{j} \\
\hat{u}_{j}=\tilde{u}_{j}+\lambda_{j} K_{j} \\
\lambda_{j}=-f_{j} / \alpha_{j-1} \\
K_{j+1}=K_{j}+v_{j} \tilde{u}_{j}
\end{array}\right\}
$$

Where $\tilde{U}=\left[\tilde{u}_{1}, \ldots, \tilde{u}_{n}\right] \hat{U}=\left[\hat{u}_{1}, \ldots, \hat{u}_{n}\right]$, and Kalman gain is given by $K=K_{n+1} / \tilde{x}_{n}$. Here $\tilde{d}$ is predicted diagonal element, and $\hat{d}_{j}$ is the updated diagonal element of the $D$ matrix. The $U-D$ filter described above is developed in ' $\mathrm{C}$ ' language and implemented in DEC Alpha computer. It has been validated using simulated trajectory data and also real flight data. A priori specification of the covariance matrices ( $Q$ and $\mathrm{R}$ ) of the process and measurement noise is necessary for tuning the $U-D$.

\section{Estimation before Modelling-Model-Based} Approach-

This approach involves two steps. In the first step, sub-optimal smoothed states of vehicle are obtained using an EUDF algorithm to perform data compatibility. This essentially makes use of the redundancy present in the measured inertial and air data variables to obtain the best state estimates from the dynamic manoeuvres. Scale factors and bias errors in the sensors are estimated by expanding the state vector to include these parameters as augmenting (additional) states and the time histories of the aerodynamic lift and drag coefficient forces corresponding to each manoeuvre are computed. In the second step, the aerodynamic parameters are estimated using the stepwise multiple linear regression (SMLR)method. For selecting an appropriate model structure, partial $\mathrm{F}$ statistics are used to build up the parameter vector by selectingsignificant parameters in the model, one at a time, until the regression equation is satisfied. In addition, at each step, the values of partial $F$, total $F$, squared multiple correlation coefficient, residual sum of squares and residual variance are evaluated. 

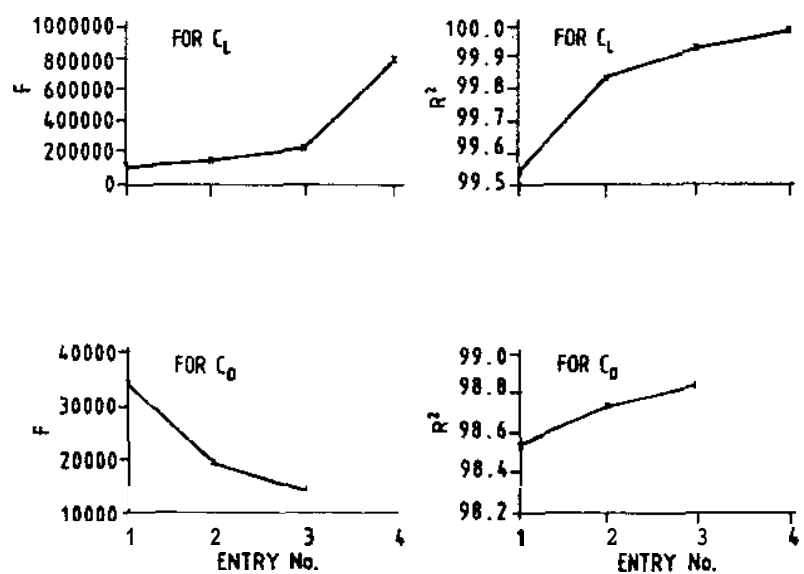

Figure 2. $\boldsymbol{F}$ and $\boldsymbol{R}^{2}$ values for lift and drag coefficients (model 1)-RC manoeuvre.

The regression equations for $C_{L}$ and $C_{D}$ (with linear terms of Taylor series) is of the form:

\subsubsection{Model 1}

$C_{L}=C_{I_{0}}+C_{I_{V}} \frac{V}{u_{0}}+C_{L_{\alpha}} \alpha+C_{L_{q}} \frac{q \bar{c}}{2 u_{0}}+C_{L_{\delta_{e}}} \delta_{e}$

$C_{D}=C_{D_{0}}+C_{D_{v}} \frac{V}{u_{0}}+C_{D_{\alpha}} \alpha+C_{D_{q}} \frac{q \bar{c}}{2 u_{0}}+C_{D_{\delta_{e}}} \delta_{e}$

The variables in model 1 are $V, a, q$, and $\delta_{e}$. These variables enter the regression equation for $C_{L}$ and $C_{D}$ in the order (based on the partial $F$ statistics) shown in Table 1. Figure 2 shows the plot of computed $F$ and $R^{2}$ versus variable entry number for a typical $R C$ manoeuvre data. As can be seen in this figure, the trend for $F$ and $R^{2}$ for lift is acceptable. However, the trend of overall $F$ for $C_{D}$ shows a decreasing trend and the $R^{2}$ value shows that this model is able to explain only $\mathbf{9 8}$ per cent of the variation in $\mathrm{C}$, This indicates the need for additional terms in the model. An additional term with $a^{2}$ as the variable is added to model 1 resulting in model $\mathbf{2}$ as given below:

\subsubsection{Model 2}

$C_{L}=C_{l_{0}}+C_{l_{i^{\prime}}} \frac{V}{u_{0}}+C_{L_{\alpha}} \alpha+C_{l_{\alpha^{2}}} \alpha^{2}+C_{l_{s}} \frac{q \bar{c}}{2 u_{0}}+C_{l_{\delta_{e}}} \delta_{e}$

$C_{D}=C_{b_{b}} \mathbf{t} C_{D_{1}} \frac{V}{u_{0}}+C_{D_{a}} \alpha \mathbf{t} C_{D_{a^{2}}} \alpha^{2} \mathbf{t} C_{D_{q}} \frac{q \bar{c}}{2 u_{0}}+C_{D_{\delta_{e}}} \delta_{e}$
Table 1. Results ofmodelstructuredeterminationusingEBM: Data set-I (RC manoeuvre)

\begin{tabular}{|c|c|c|c|c|}
\hline $\begin{array}{c}\text { Variable } \\
\text { entered } \\
\text { No. Name }\end{array}$ & \multicolumn{2}{|c|}{$\begin{array}{cc}\begin{array}{cc}\text { Relating } & R^{2} \\
\text { derivative } & (\%)\end{array} \\
\end{array}$} & Overall $F$ & \multirow[t]{2}{*}{$\begin{array}{c}\text { Partial } \\
\text { F statistics }\end{array}$} \\
\hline \multicolumn{4}{|c|}{ Lift parameters: Model I } & \\
\hline I $a$ & $C_{l_{\alpha}}$ & 99.5450 & $0.1089 \mathrm{E}$ & 0.5 \\
\hline $2 V$ & $C_{L_{r} .}$ & 99.8320 & $0.1476 \mathrm{E}+06$ & $0.1316 \mathrm{E}+02$ \\
\hline $3 \alpha_{e}$ & $C_{L_{\text {s. }}}$ & 99.9285 & $0.2310 \mathrm{E}+06$ & $0.6641 E+02$ \\
\hline $4 q$ & $C_{l_{q}}$ & 99.9842 & $0.7852 E+06$ & $0.4186 \mathrm{E}+02$ \\
\hline \multicolumn{5}{|c|}{ Drag parameters: Model I } \\
\hline I $a$ & $C_{p_{\alpha}}$ & 98.5372 & $0.3355 E+05$ & $0.9379 E+02$ \\
\hline $2 V$ & $C_{D_{r}}$ & 98.7239 & $0.1923 \mathrm{E}+05$ & $0.9502 \mathrm{E}+0$ \\
\hline $3 \delta_{e}$ & $C_{D_{\delta_{\varepsilon}}}$ & 98.8348 & $0.1402 E+05$ & $0.6869 \mathrm{E}+01$ \\
\hline $4 q$ & $C_{D_{a}}$ & & Not Enler & \\
\hline \\
\hline I $a$ & $C_{l_{\alpha}}$ & 99.5450 & $0.1089 \mathrm{E}+06$ & $0.1699 E+03$ \\
\hline $2 V$ & $C_{l_{v}}$ & 99.8320 & $0.1476 \mathrm{E}+06$ & $0.1776 \mathrm{E}+02$ \\
\hline $3 \delta_{e}$ & $C_{L_{\delta_{\mathrm{e}}}}$ & 99.9285 & $0.2310 \mathrm{E}+06$ & 0.1380 \\
\hline $4 \mathrm{q}$ & $C_{L_{q}}$ & 99.9842 & $0.7852 \mathrm{E}+06$ & $0.9524 \mathrm{E}+02$ \\
\hline $5 a^{\prime}$ & $C_{I_{a_{2}}}$ & 99.9967 & $0.2950 \mathrm{E}$ t 07 & 0.427 \\
\hline \multicolumn{5}{|c|}{ Drag parameters: Model 2} \\
\hline I $\alpha^{2}$ & $C_{D_{a^{2}}}$ & 99.6417 & $0.1385 E+06$ & $0.1288 \mathrm{E}+03$ \\
\hline $2 \alpha$ & $C_{D_{a}}$ & 44 & $0.2147 \mathrm{E}+06$ & $-0.5696 \mathrm{E}+02$ \\
\hline $3 q$ & $C_{D_{q}}$ & 99.9234 & $0.2155 \mathrm{E}+06$ & $0.2219 E+02$ \\
\hline $4 \delta_{e}$ & $C_{D_{\delta_{\mathrm{e}}}}$ & 99.9614 & $0.3208 \mathrm{E}+06$ & $0.2499 E+02$ \\
\hline $5 V$ & $C_{D_{v}}$ & 99.9664 & $0.2937 E+06$ & $-0.8513 E+01$ \\
\hline
\end{tabular}

The order in which the variables enter the regression equation is shown in Table 1 and the results of $F$ and $\boldsymbol{R}^{2}$ are plotted in Fig. 3. The trends indicate the adequacy of this model for $C_{D}$, although the improvement in $\mathrm{C}$, is only marginal. Hence $\alpha^{2}$ term is included only in drag equation and this structure is used for $C_{1}$ and $C_{b}$ in the MBAs. Subsequently, the drag polars can be reconstructed using the estimated parameters in the Taylor series representation of the aerodynamic coefficients'. The advantage of using this method is that the model structure can be determined and used in 
other techniques for further refinement. The method does not require initial values of the unknown parameters to start the estimation procedure (due to one-shot nature of the regression in the second step).

\section{$2.4 U$-D Filter-Non-Model-Based Approach}

This is a NMBA in which the aerodynamic derivatives are not estimated/required. As in EBM method, using kinematic consistency checking procedure, the aircraft states are reconstructed by EUDF. Using the filtered states (and the kinematic relations), the dimensional forces $X$ and $Z$ are computed. A centrally pivoted five-point algorithm for numerical differentiation obtains the time derivatives required at this stage.

Next, the time histories of the nondimensional aerodynamic coefficients $C_{x}$ and $C_{z}$ are computed using the aircraft mass characteristics. The drag and lift coefficients (time histories) $C_{L}$ and $C_{D}$ are obtained and the drag polar determined. The kinematic consistency equations ${ }^{6}$ are:

\subsubsection{State Equations}

$$
\left.\begin{array}{rlrl}
\dot{u}= & -(q-\Delta q) w+(\mathrm{r}-\Delta r) v & \\
& -g \sin \theta+\left(a_{x}-\Delta a_{x}\right), & u(0)=u_{0} \\
v= & -(\mathrm{r}-\Delta r) u+(\mathrm{p}-\mathrm{A} p) w \\
& +g \cos \theta \sin \phi+\left(a_{x}-\Delta a_{y}\right), \quad v(0)=v_{0} \\
\dot{w}= & -(p-\Delta p) v+(q-\Delta q) u & \\
& +g \cos \theta \cos \phi+\left(a_{x}-\Delta a_{z}\right), & w(0)=w_{0} \\
\dot{\phi}= & -(p-\Delta p)+(q-\Delta q) \sin \phi \tan \theta \\
& +(\mathrm{r}-\Delta r) \cos \phi \tan \theta, & \phi(0)=\phi_{0} \\
\dot{\theta}= & (q-\Delta q) \cos \phi-(r-\Delta r) & \\
& & \sin \phi, & \theta(0)=\theta_{0} \\
\dot{\psi}= & (q-\Delta q) \sin \phi \sec \theta & \\
& +(r-\Delta r) \cos \phi \sec \theta, & \psi(0)=\psi_{0} \\
\dot{h}= & u \sin \theta-v \cos \theta \sin \phi & \\
& -w \cos \theta \cos \phi, & h(0)=h_{0}
\end{array}\right)
$$
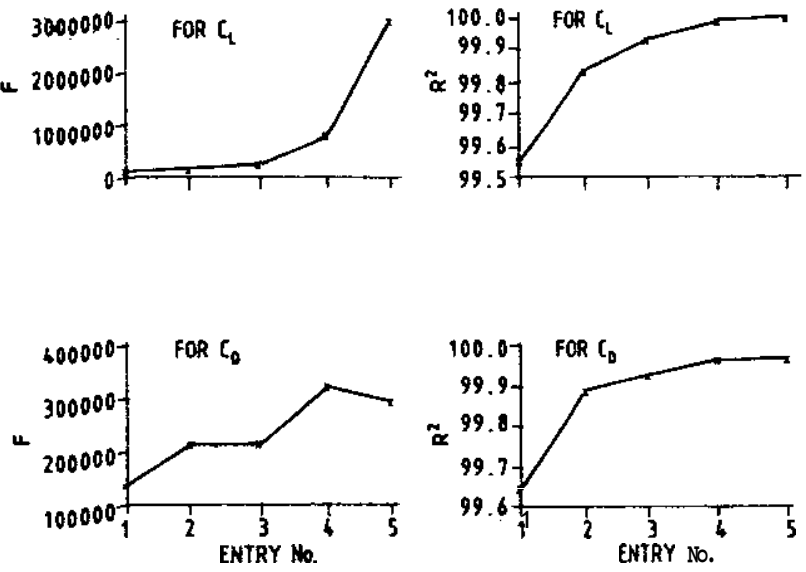

Figure 3. $F$ and $R^{2}$ values for lift and drag Coefficients (model 2)-RC mano:uvre.

\subsubsection{Observation Equations}

$$
\begin{aligned}
& V_{m}=\sqrt{u_{n}^{2}+v_{n}^{2}+w_{n}^{2}}+\Delta V \\
& \alpha_{m}=K_{\alpha} \tan ^{-1}\left(\frac{w_{n}}{u_{n}}\right)+\Delta \alpha \\
& \beta_{m}=\sin ^{-1}\left(\frac{v_{n}}{V_{m}}\right) \phi_{m}=\phi \\
& \theta_{m}=K_{\theta} \theta+\Delta \theta \psi_{m}=\psi \\
& h_{m}=h
\end{aligned}
$$

where $u_{n}, v_{n}$ and $w_{n}$ are the velocity components along three 'axes at the nose boom and they are given by

$$
\left.\begin{array}{l}
u_{n}=u-(r-\Delta r) y_{n} \mathrm{t}(q-\Delta q) z_{n} \\
v_{n}=y-(p-\Delta p) z_{n} \mathrm{t}(r-\Delta r) x_{n} \\
w_{n}=w_{-}-(q-\Delta q) x_{n}+(p-\Delta p) y_{n}
\end{array}\right\}
$$

where $\boldsymbol{x}_{n}, \boldsymbol{y}_{n}$ and $z_{n}$ are the nose boom offset distances from the center of gravity. Using the corrected states and linear accelerations in the following equations, the lift and drag coefficients can be estimated:

$$
\left.\begin{array}{l}
a_{x}=\frac{\bar{q} S}{m}\left(C_{X}\right)+\frac{F_{e}}{m} \cos \sigma_{T} \\
a_{x}=\frac{\bar{q} S}{m}\left(C_{z}\right)-\frac{F_{e}}{m} \sin \sigma_{T}
\end{array}\right\}
$$


The lift and drag coefficients are computed from $C_{X}$ and $C_{z}$ using following equations:

$$
\left.\begin{array}{l}
C_{L}=-C_{Z} \cos a+C_{X} \sin \alpha \\
C_{D}=-C_{X} \cos \alpha-C_{Z} \sin a
\end{array}\right\}
$$

\subsection{U-D Filter-based Mocl-BasedApproach}

This is a MBA where the mathematical model (Taylor series expansion of the aerodynamic coefficients) is formulated with unknown system parameters as augmented statesand hence the parameter estimation problem is transformed into a stateestimation problem leading to EUDF.

A priori specification of the covariance matrices of the process and measurement noise is necessary to use the EUDF. The mathematical model formulation is as follows:

\subsubsection{State Model}

$$
\begin{aligned}
& \dot{V}=-\frac{\bar{q} S}{m}\left(C_{D_{0}}+C_{D_{v}} \frac{V}{u_{0}}+C_{D_{\alpha}} \alpha+C_{D_{\alpha^{2}}} \alpha^{2}\right. \\
& \left.+C_{D_{q}} \frac{q \bar{c}}{2 u_{0}}+C_{D_{b_{e}}} \delta_{e}\right)+\frac{F_{e}}{m} \cos \left(\alpha+\sigma_{T}\right) \\
& \mathbf{t} g \sin (\alpha-\theta)+b_{w} \\
& \dot{\alpha}=-\frac{\bar{q} S}{m V}\left(C_{L_{0}}+C_{L_{v}} \frac{V}{u_{0}}+C_{L_{u}} \alpha\right. \\
& \left.+C_{L_{\varphi}} \frac{q \bar{c}}{2 u_{0}}+C_{L_{\delta_{e}}} \delta_{e}\right)-\frac{F_{e}}{m V} \sin \left(\alpha+\sigma_{T}\right)+q \\
& t \underset{V}{g} \operatorname{sg}(\alpha-\theta)+b \\
& \theta=q \mathbf{t} b_{t h}
\end{aligned}
$$

where $b_{p,}, b_{s i}$ and $b_{t h}$ are the bias parameters in state equations. In addition to the above state model, all the unknown parameters in the state and observation equations are augmented as state vectors.

\subsubsection{Observation Model}

$$
\begin{aligned}
& V_{m}=V \\
& a_{, 1}=\mathrm{a} \\
& \theta_{m}=\theta \\
& a_{x_{m}}=\frac{\bar{q} S}{m}\left(C_{X}\right)+\frac{F_{e}}{m} \cos \sigma_{T} \\
& a_{-m}=\frac{\bar{q} S \cdot\left(C_{Z}\right)-\frac{F_{e}}{m}}{\frac{\bar{m}}{m}} \sin \sigma_{T}
\end{aligned}
$$

with

$$
\begin{aligned}
C_{Z}= & -C_{L} \cos a-C_{D} \sin a \\
C_{X}= & C_{L} \sin \mathrm{a}-C_{D} \cos a \\
C_{L}= & C_{L_{0}}+C_{L_{V}} \frac{V}{u_{0}}+C_{L_{\alpha}} \alpha+C_{L_{q}} \frac{q \bar{c}}{2 u_{0}}+C_{L_{\delta_{e}}} \delta_{e} \\
C_{D}= & C_{D_{0}}+C_{D_{V}} \frac{V}{u_{0}}+C_{D_{\alpha}} \alpha+C_{D_{\alpha^{2}}} \alpha^{2} \\
& +C_{D_{q}} \frac{q \bar{c}}{2 u_{0}}+C_{D_{\delta_{e}}} \delta_{e}
\end{aligned}
$$

The lift and drag polars are determined using the estimated augmented state parameters in Eqn (I 5).

\subsection{Stabilised Output Error Method}

The output error method' (OEM) is the most widely used technique for the estimation of parameters of stable dynamical systems. It has been successfully utilised for the estimation of stability and control derivatives of aircraft from flight data. However, the method poses severe difficulties when applied to parameter estimationfor fly-by-wire (FBW) aircraft. When the basic aircraft is unstable, numerical integration of the state model leads to diverging solutions. This instability caused due to numerical divergence can be overcome by incorporating stabilisation into the OEM usingmeasured states for those aerodynamic derivatives, which cause instability in the system model. While this approach has the advantage of stabilising the system, it needs accurate measurement of states. For the aerodynamic coefficients, the model structure selected using EBM (Fig. I) can be used. The state space mathematical model is formulated with three $(\mathrm{V}$, a and $\theta)$ states and five $(V, \alpha, \theta, a$, and $a$, ) observable variables. The unknown parameters in the state and observation equations 
are estimated iteratively and these are used to construct the lift and dragpolars. The mathematical formulation for stablised output error method (SOEM) is the same as in Eqns (13)to ( I 5) except that the measured states $a$ and $q$ are used for artificial stabilisation of numerical integration of the state equations, i.e. Eqn (13).

\subsection{Extended Forgetting Factor Recursive}

\section{Least Squanes Method}

An effective recursive method called extended forgetting factor recursive least squares (EFFRLS) method for the estimation of drag polar is described. This method does not require knowledge of process and measurement noise statistics. It only requires a suitable choice of a forgetting factor*(FF). The main advantage of this method is that only one adjustable parameter is required compared to several elements of $\mathrm{Q}$ and $R$ required for tuning of a Kalman filter. The algorithm used in the non-model based mode ${ }^{8}$ is given as

$$
\begin{aligned}
& x_{k+1 j}= \Phi_{k} x_{k j} \\
& x_{k+1 / j+1}=\Phi_{k}\left[L_{k / j}\left(y_{j+1}-H_{j+1} \Phi_{j+1 / k} x_{k / j}\right)\right] \\
& L_{k^{\prime} j}=P_{k / j} \Phi_{j+1 / k}^{\prime} H_{j+1}^{\prime}\left(\lambda I+H_{j+1} \Phi_{j+1 / k} P_{k / j} \Phi_{j+1 / k}^{\prime} H_{j+1}^{\prime}\right)^{-1} \\
& P_{k+1 / j+1} \equiv\left(X_{k+1 / j+1} \Lambda_{j+1} X_{k+1 / j+1}\right)^{-1} \\
& \quad \equiv \lambda^{-1} \Phi_{k}\left[I-L_{k / j} H_{j+1} \Phi_{j+1 / k} P_{k^{\prime} j} \Phi_{k}^{\prime}\right.
\end{aligned}
$$

FF, A should be very close to l but less than 1. If $\mathrm{FF}$ is equal to 1 , then it gives ordinary least squares solution. The memory index (MI) of the filter can be defined as $M I=1 /(1-F F)$. Thus if $\mathrm{FF}=\mathbf{I}$, then $\mathrm{MI}$ is infinity - thefilter is said to have infinite memory. This means that the entire data set is given equal weighing. If $\mathrm{FF}>\mathrm{I}$, the MI will also be smaller (finite memory). thereby implying that the past data are given less weighting, since the weighting factor used in the least squares perforniance functional is given as

$$
\lambda^{k-i} ; i=1,2, \ldots, k
$$

Choice of FF is based on the following considerations. If the process noise variance is expected to be large, the FF should be small, since the past data is not giving more information on the current state/parameter. If the process noise variance is relatively smaller than the measurement noise variance, the FF should be of a large value. This implies that more data should be used to average out the effect of the noise on measurements. FF can also be linked to the column rank of the observation model $\mathrm{H}$. lfthis rank is larger, there is more information (contained by the $k^{\text {th }}$ measurement data) on the present state. FF can also be taken as inversely proportional to the condition number of the data matrix:

$$
P_{k j}=\left(X_{k j}^{\prime}, X_{k j}\right)^{-1}
$$

If the condition number of the matrix is large, then one would like to give less emphasis on the past data, and hence the FF should be smaller. The condition number of a matrix is defined as the ratio of magnitude of the largest eigenvalue to the magnitude of the smallest eigenvalue. The above are general guidelines to choose a FF. For a given application specific evaluation study is generally required to arrive at a suitable FF. Thus the FF may be chosen as

$$
\begin{aligned}
& \lambda \propto \frac{\operatorname{Variance}(R)}{\operatorname{Variance}(Q) \text { Condition No.(data matrix } P)} \\
& \overline{\text { Column rank (fi) }}
\end{aligned}
$$

From the above it is clear that the weighting factor is intended to ensure that data in the distant past are forgotten in order to afford the possibility of following the statistical variation of the measurement data.

\section{RESULTS \& DISCUSSIONS}

Aircraft responses are generated at three representative flight conditions from the FBW aircraft simulator using two dynamic performance manoeuvres as 
DEF SCI J, VOL 51, NO 1, JANUARY $200 \mathrm{l}$

Table 2. Percentage fit error for lift and drag coeficients

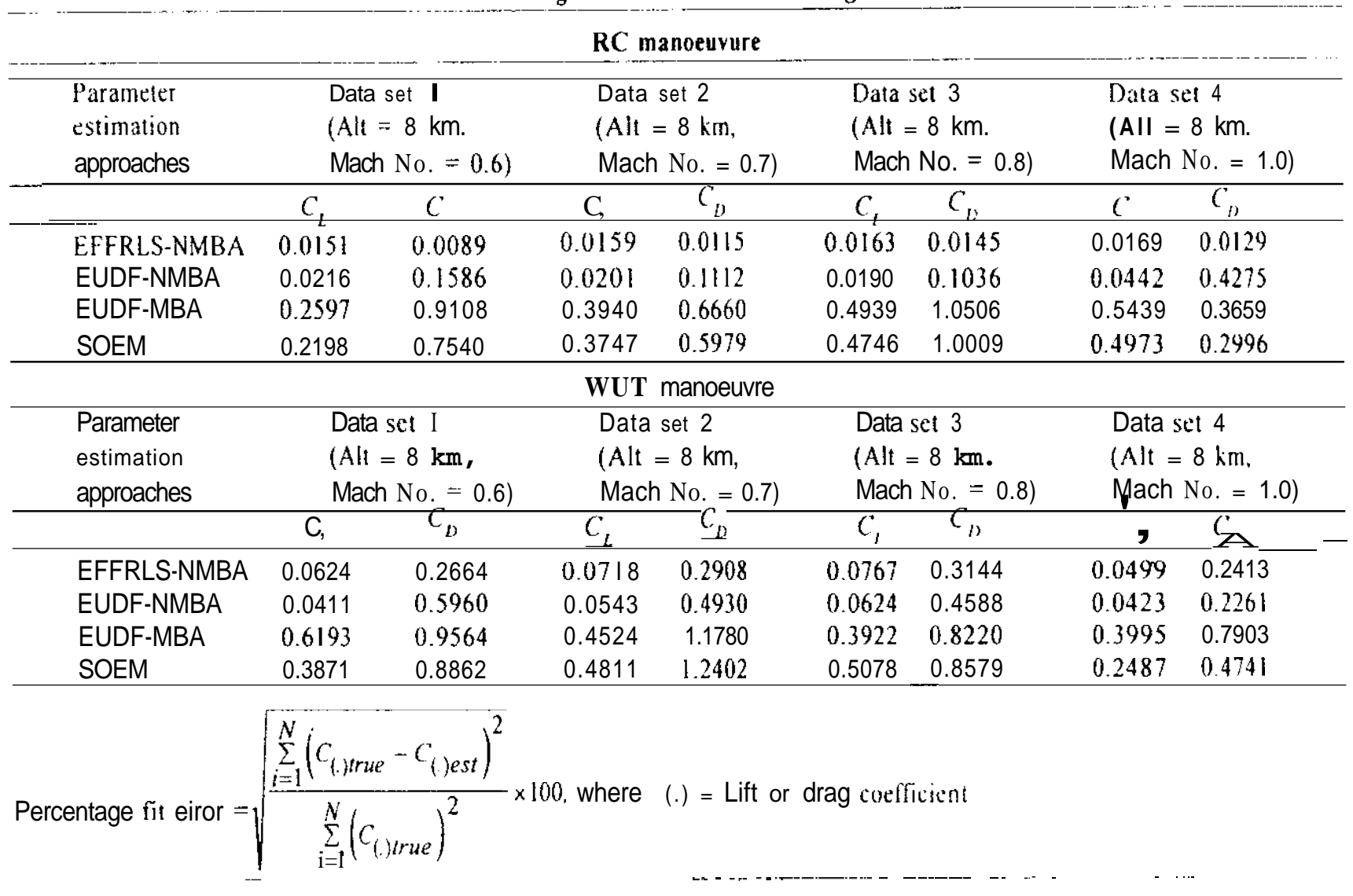

\section{Roller Coaster}

Roller coaster ( $\mathrm{RC}$ ) longitudinal manoeuvres are generated for which the simulated vehicle is taken through $1 \mathrm{~g}-2 \mathrm{~g}-0 \mathrm{~g}-\mathrm{g}$ normal acceleration cycle at the rate of $0.1 \mathrm{~g} / \mathrm{s}$ for Mach Nos. $=0.6,0.7,0.8$ and 1.0 at altitude $=8 \mathrm{~km}$.

\section{Wind-up-Turn}

Wind-up-turn (WUT) coupled manoeuvre data are generated for which the vehicle is progressively banked and loaded so that the $g$ linearly builds up from I $g$ to nearly maximum $g$, and angle of attack ranges from $5^{\circ}$ to $20^{\circ}$, at the same flight conditions as in $\mathrm{RC}$ manoeuvre.

The $\mathrm{RC}$ manoeuvres are generated from the $\mathrm{FBW}$ aircraft simulator, while it is operating in the hatch simulation mode. WUT data is generated by actually flying the simulator by a pilot/engineer.

Table 2 gives the fit error performance of different methods at various flight conditions for wo types $\because$ manoemres. It can he seen that the EFFRLSGMBA and EUDF-NMBA Dorform better than the other tw o MBAs. Drag polars results obtained from $\mathrm{RC}$ and WUT manoeuvre at altitude $8 \mathrm{~km}$ and Mach No. 0.6 are presented in Figs 4 and 5, respectively, for the four methods. It is seen that the results are satisfactory. Though more results have been generated at other flight conditions, but for the sake of brevity, these have not been included here.

The SOFM is an iterative process and hence requires more time for drag polar determination, EUDF is a recursive process and could he an attractive alternative to the SOEM. However, it requires proper choice of the process and measurement noise statistics. The two-step method EBM that helps in model selection based on statistical criteria is a good method for drag polar determination. Houever, it could be more time consuming. It is included here only for the sake of completion and to show the link with other methods. AN M B A could be preferred over MBA. as it would require less computation time and would still give accurate results lor drag polars from Ilight data. It is also a potential candidate for real-tiiiie on-line determination of drag pqlars. This approacli has recently been validated 4 sing the data from transport category aircraft. It is being 

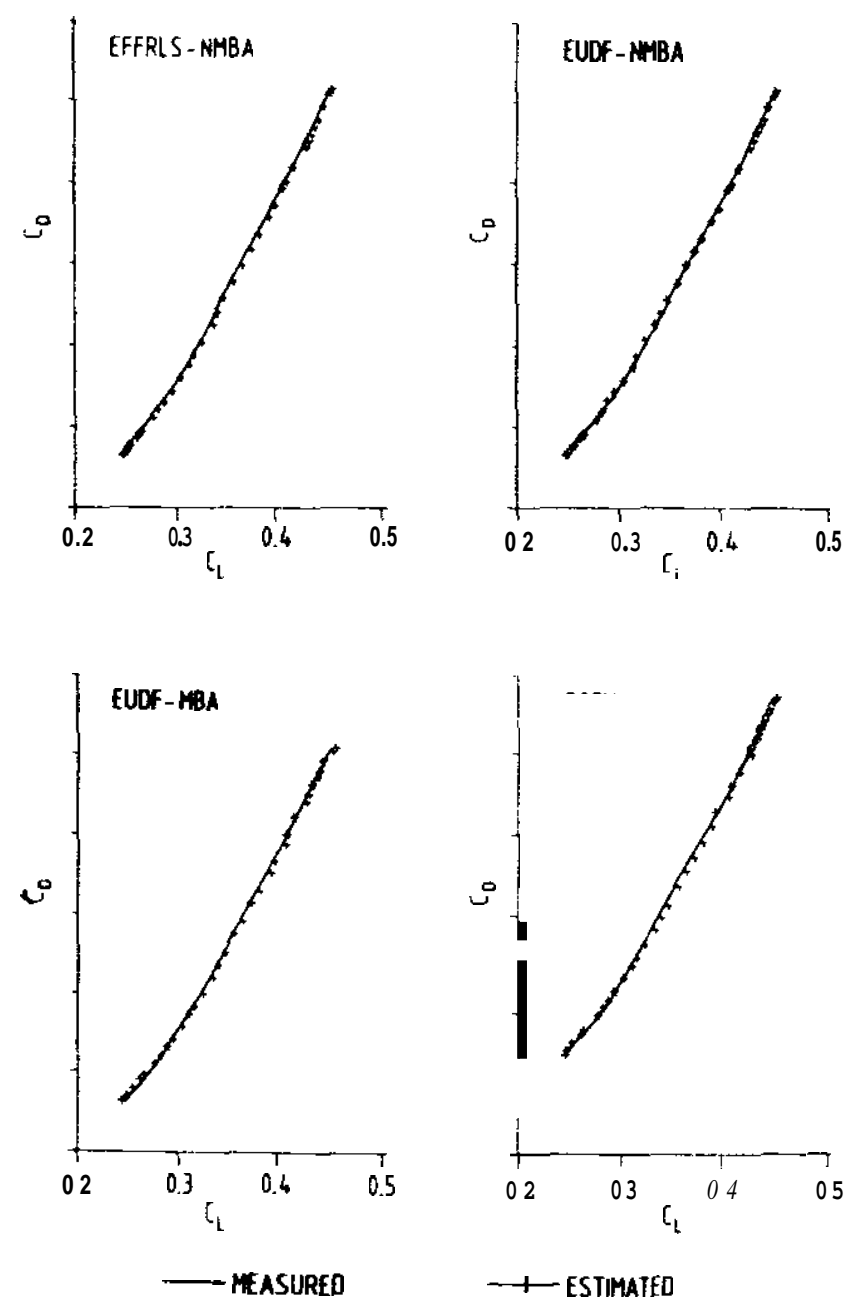

- ESTIMAIFD

Figure 4. Drag polars - roller coaster manoeuvre (data set 1).

further studied for in-flight drag polar determination.

For the upcoming flights of an FBW aircraft the approaches evaluated here are planned to be used for the determination of drag polars using real flight test data.

\section{CONCLUSION}

Mainly four parameter/state estimation methods have been evaluated for the determination of drag polar from dynamic performance manoeuvre data for an FBW aircraft. White it is posible to get very good estimates of drag polsrs from all the methods, the NMBAs are very efficient and less time consuming. They can also he applied for real time estimation of drag polars from flight data. A novel approach for estimation of drag polars has been validated. The latter requires 10 choose only one adjustable factor compared to several (as in Kalman filter cases). It is very promising method (or nn-line determination of drag polars from real
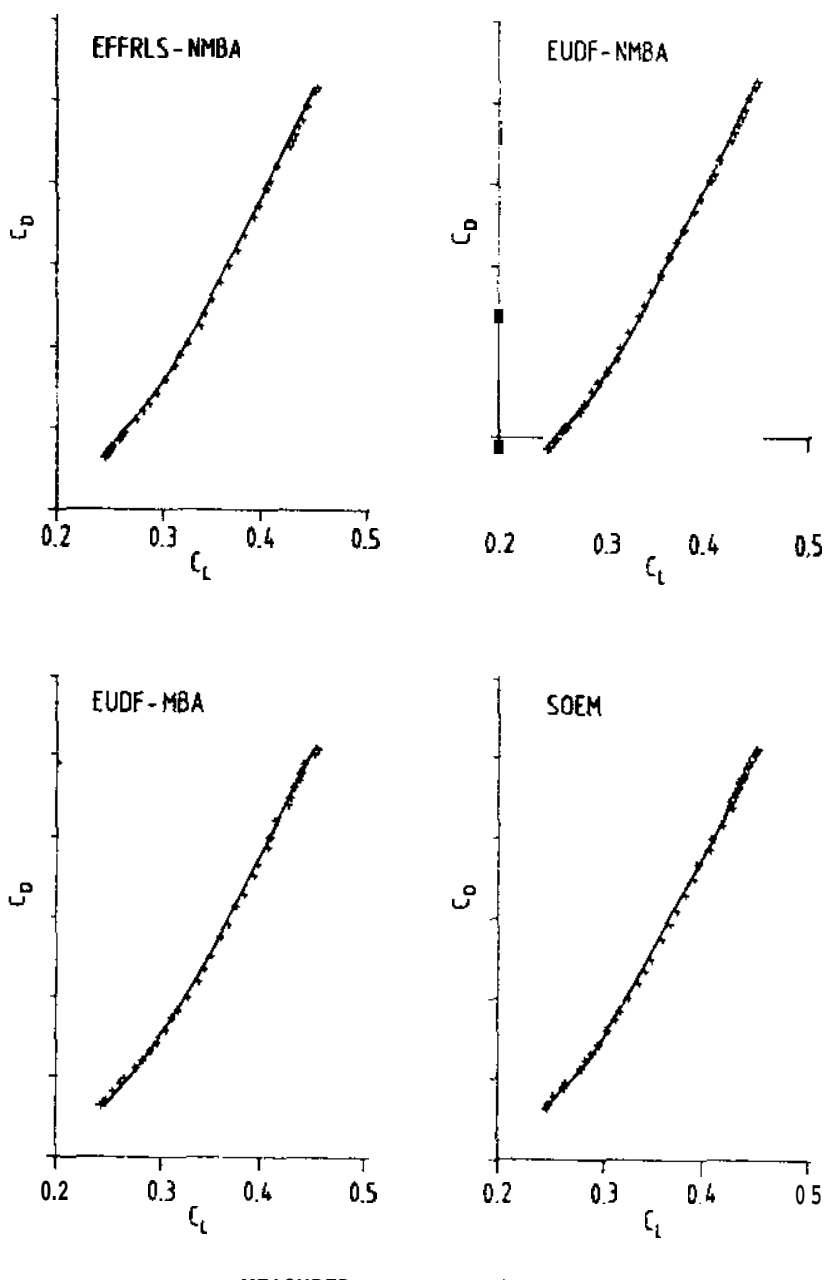

Figure 5. Drag polars - wind-up-turn manoeuvre (data set 1)

flight-test data.

\section{ACKNOWLEDGEMENT}

Thanks are due to Dr T.G. Pai, Project Director, (Technology Development, Aeronautical Development Agency) for permission to publish the work.

\section{REFERENCES}

1. Knaus, A. A technique to determine lift and drag polars in flight Journal of Aircraft, 1983, 20, (7), 587-93.

2. Girija,G.; Parameswaran, V.; Raol, J.R. \& Balakrishna, S.; Estimation of aircraft performance and stability characteristics by dynamic manoeuvres using maximum likelibood estimation techniques. In Pre-prints of 9 th $^{\mathrm{t}}$ IFACIIFORS Symposium on Identification and System Parameter Estimation 8-12 July 1991, Budapest. Hungary. 
3. Girija. G.: \& Raol, J.R. Analysis of stabilized output error methods. IEE Proc. Control Theory Appl., 1596, 143, (2), 209-16.

4. Girija, G. \& Rao, J.R. Estimation of aerodynamic parameters from dynamic manoeuvres using estimation before modelling procedure. Aero. Soc. India, 1996, 48.

5. Bierman, G.J. Factorisation methods for discrete sequential estimation. Academic Press, New York, 1977.

6. Girija, G. \& Raol, J.R. PC-based flight path reconstruction using UD factorization filtering algorithm. Def. Sci. J., 1993, 43, (4), 429.47.
7. Maine, R.E. \& lliff, K.W.. Application of parameter estimation to aircraft stability and control-output error approach. National Aeronautics \& Space Administration. USA. 1986. NASA-RP-1168.

8. Zhu, Y. Efficient recursive state estimator for dynamic systems without knowledge of noise covariances. IEEE Trans., 1999, AES, 35 (1), 102-13.

9. Girija, G.; Basappa; Raol. J. R. \& Madhuranath, Padma. Evaluation of methods for determination of drag polars of unstable/augmented aircraft. Paper presented at the 38th Aerospace Sciences Meeting \& Exhibit, [0-!3 January 2000. Reno, NV, USA. Paper No. AIAA-2000-0501. 